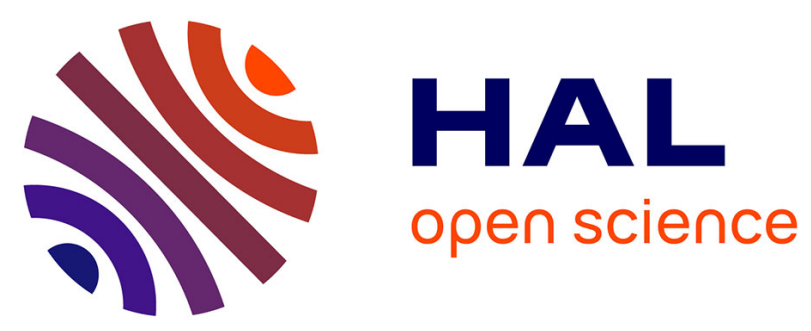

\title{
Apport de la méthode ICP-MS couplée à l'ablation laser pour la caractérisation des archéomatériaux
}

Bernard Gratuze, Alain Giovagnoli, Jean-Noël Barrandon, Philippe Telouk, Jean-Louis Imbert

\section{- To cite this version:}

Bernard Gratuze, Alain Giovagnoli, Jean-Noël Barrandon, Philippe Telouk, Jean-Louis Imbert. Apport de la méthode ICP-MS couplée à l'ablation laser pour la caractérisation des archéomatériaux. Archeosciences, revue d'Archéométrie, 1993, 17, pp.89-104. 10.3406/arsci.1993.908 . hal-01829851

\section{HAL Id: hal-01829851 https://hal.science/hal-01829851}

Submitted on 5 Jul 2018

HAL is a multi-disciplinary open access archive for the deposit and dissemination of scientific research documents, whether they are published or not. The documents may come from teaching and research institutions in France or abroad, or from public or private research centers.
L'archive ouverte pluridisciplinaire HAL, est destinée au dépôt et à la diffusion de documents scientifiques de niveau recherche, publiés ou non, émanant des établissements d'enseignement et de recherche français ou étrangers, des laboratoires publics ou privés. 


\title{
APPORT DE LA METHODE ICP-MS COUPLEE A L'ABLATION LASER POUR LA CARACTERISATION DES ARCHEOMATERIAUX
}

\author{
Bernard GRATUZE*, Alain GIOVAGNOLI, Jean Noël BARRANDON*, Philippe TELOUK** \\ et Jean Louis IMBERT**
}

\begin{abstract}
Résumé: Au cours des années quatre-vingts, de nouvelles méthodes d'analyses ont été développées. Parmi celles-ci, l'ICP-MS semble pouvoir apporter une aide précieuse à la caractérisation des maté riauxarchéologiques. Le couplagé récent de cette methode avec l'ablation laserpermet d'autre part d'envisagerl'application de cette technique lorsque
l'objet à analyser ne peut être échantillonné.

Après avoir décrit rapidement le principe de l'ICP-MS (Inductively Coupled Plasma Mass Spectrometry), nous
étudierons l'intérêt du couplage de cette méthode avec l'ablation Nous présenterons ensuite les cette méthode avec l'ablation laser.

d'Analyse du CNRSà Solaize. Au cours de ce travail obtenus lors d'une étude conjointe réalisée au Service Central et des matières siliceuses (verre et obsidienne) ont été étudiés. Nous nous sommes plus particulièrement attachés à vérifiés.

- le caractère non-destructif de la méthode;

résultats obtenus par cette méthode et ceux obtenus lats. Pour cela, une comparaison a été effectuée entre les nucléaire développées au Centre $\mathrm{E}$. Babelon pour ces matériaux;

- les limites de détection de la méthode;

- les éléments détectés par rapport aux problématiques archéologiques envisagées.
\end{abstract}

Abstract: Inductively coupled plasma mass spectrometry (ICP-MS) is a new analytical method developed during the eighties, its recent association with laser ablation (LA-ICP-MS) gives to the analyst a powerful tool. In this work, we study the potentiality of this technique to characterise "non destructively" various archaeological materials such as d'Analyse of the CNRS in Solaize are published here. Depending on the material, we focus mainly oncre.

- non destructivity;

- non destructivity;

- precision and accuracy, a comparison is made between the results obtained with LA-ICP-MS and those obtained using a cyclotron FNAA and protons activation analysis PAA). Centre E. Babelon (fast neutrons activation analysis - sensitivity;

- the help bring by the results obtained with this method to solve archaeological problems.

Mots-clés: Ablation laser, plasma, spectrométrie de masse, analyse par activation, verre, métaux, archéomatériaux.

Key-words: Laser ablation, plasma, mass spectrometry, activation analysis, glass, metals, archaeological materials.

\section{INTRODUCTION}

Les méthodes d'analyse globale et non destructive d'objets archéologiques sont peu nombreuses. Parmi celles-ci, les méthodes comme la fluorescence $X$ ou le PIXE donnent des résultats fiables uniquement lorsque l'objet est plan et non corrodé, ce qui n'est pas le cas de la plupart des objets archéologiques. D'autre part, en analyse non destructive, ces méthodes ne permettent pas le dosage des éléments traces sans lesquels certaines problématiques archéologiques ne peuvent être réso-
Depuis une quinzaine d'années, le Centre E. Babelon développe, autour d'un accélérateur de particules (cyclotron), l'application des méthodes d'analyse par activation nucléaire afin de pouvoir répondre aux problématiques archéologiques nécessitant des analyses élémentaires sans toutefois détruire ou détériorer les objets par un prélèvement. Si ces méthodes ont montré leur fiabilité et leur intérêt, elles ont toutefois deux inconvénients majeurs:

- le coût important des moyens mis en oeuvre;

- la durée importante des analyses (entre quelques jours et deux mois): si l'on veut d'une part minimiser la 
radioactivité créée dans l'objet et d'autre part avoir une grande sensibilité dans la détection des éléments traces.

Cette durée importante des temps d'analyse limite le nombre d'échantillons analysés et par là même, l'utilisation de nos méthodes pour résoudre certaines problématiques archéologiques nécessitant un nombre important d'analyses. La recherche de nouvelles méthodes d'analyses non destructives ou quasiment non destructives, nous permettant d'élargir nos champs d'application vers ces problématiques, fait partie de nos préoccupations.

Au milieu des années soixante-dix, les premiers appareils utilisant un plasma d'argon induit par haute fréquence comme source d'atomisation et d'excitation avec une détection par spectrométrie optique (ICPAES: inductively coupled plasma atomic emission spectrometry) ont été commercialisés. Le plasma étant aussi une source d'ions, la détection de ces ions par spectrométrie de masse a été rapidement développée et les premiers appareils utilisant le couplage plasma-spectrométrie de masse (ICP-MS) sont apparus au début des années quatre-vingts. Si l'échantillon est généralement introduit dans le plasma sous la forme d'un aérosol obtenu à partir d'une solution aqueuse, les méthodes d'introduction directe de solides ont semblé rapidement attrayantes. Ces méthodes se divisent en deux catégories (Gray, 1985; Houk, 1986): l'introduction directe de l'échantillon massif dans le plasma et l'introduction d'un nuage de particules créé à partir du solide, soit au moyen d'un arc électrique, soit au moyen d'un faisceau laser (ablation laser: LA-ICP-MS).

Ce sont les potentialités de caractérisation «non destructive» des archéomatériaux offertes par cette dernière méthode qui ont été étudiées au cours de ce travail réalisé au Service Central d'Analyse du C.N.R.S. à Solaize.

Cette étude a été effectuée sur des métaux précieux (fragments de pièces d'or et d'argent) et des matières siliceuses (verre et obsidienne). D'autres matériaux comme les objets cuivreux ont été rejetés en raison des phénomènes de corrosion et des hétérogénéités de composition (précipités de plomb) qui y sont souvent observés.

Les résultats obtenus par LA-ICP-MS ont été comparés à ceux obtenus sur les mêmes objets avec les méthodes développées et utilisées par le Centre E. Babelon: activation protonique (PAA) pour l'or et l'argent (Barrandon, 1980), activation avec des neutrons rapides de cyclotron (FNAA) pour le verre et l'obsidienne (Gratuze, Barrandon et al., 1992). Nous nous sommes plus particulièrement attachés à vérifier les points suivants:

- le caractère non destructif de la méthode. Bien que cette méthode ne soit pas réellement non destructive un micro-prélèvemént étant effectué par l'ablation laser - nous avons essayé de réduire à son minimum l'impact laser produit sur l'objet. Le qualificatif de non destructif peut en effet être attribué à une méthode si le prélèvement reste invisible à l'oeil nu ainsi qu'à l'observation avec une loupe de faible grossissement (x 10);

- la reproductibilité et la représentativité des résultats. La réduction de la taille de l'impact laser peut avoir des conséquences sur la reproductibilité des résultats. En effet, l'homogénéité des différents matériaux peut ne

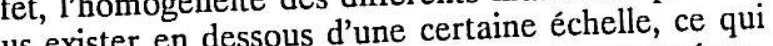
implique que les résultats obtenus ne sont pas représentatifs de la composition réelle de l'objet. Nous nous attacherons à vérifier la reproductibilité du signal obtenu entre plusieurs impacts effectués à différents endroits d'un même objet et nous comparerons les valeurs trouvées par LA-ICP-MS à celles obtenues par les méthodes d'analyse par activation qui permettent une analyse globale de l'échantillon;

- les limites de détection de la méthode. Une comparaison des limites de détection des différentes méthodes a été effectuée;

- les éléments détectés par rapport aux problématiques archéologiques envisagées. Cette méthode permet l'analyse d'un nombre important d'éléments qui ne sont pas détectés par les méthodes d'analyse par activation développées au Centre E. Babelon. Les objets soumis à l'analyse ont été sélectionnés en fonction des problématiques posées et une analyse minutieuse des éléments détectés par ICP-MS sera effectuée afin de savoir si ces éléments mettent en évidence des phénomènes nouveaux ou aident à résoudre les questions posées.

\section{MATERIEL ET METHODE}

Le couplage plasma induit par hautes fréquences, spectrométrie de masse et ablation laser fait appel à trois technologies différentes (Jarvis et al., 1992) (fig. 1):

\section{LES LASERS}

Le laser sert à pulvériser l'échantillon (Arrowsmith, 1987). La vapeur produite, constituée en grande partie de gaz monoatomiques, est entraînée vers la torche à plasma par un courant d'argon. Dans notre cas, le laser utilisé, de type Néodyme YAG, délivre un faisceau infra rouge de longueur d'onde $1064 \mathrm{~nm}$. La lampe flash excitatrice est une lampe au xénon. Le faisceau laser est réfléchi à $90^{\circ}$ sur un miroir et traverse un système optique qui le focalise sur l'échantillon placé dans une cellule en quartz balayée par un courant d'argon. Dans des conditions normales, le diamètre de l'impact est de l'ordre de 50 à $200 \mu \mathrm{m}$ en fonction de la dureté du matériau, ce qui est supérieur au pouvoir de résolution de l'oeil (environ $100 \mu \mathrm{m}$ ). Des modifications du système optique ont donc été apportées afin d'obtenir un diamètre d'impact compris entre 30 et $60 \mu \mathrm{m}$, ce qui le rend invisible à l'oeil nu. Une mesure du taux de transfert a été réalisée par ablation d'un échantillon rendu radioactif.

\section{LES PLASMAS INDUTTS PAR HAUTES FREQUEN- CES}

Le plasma est une excellente source d'ionisation grâce à l'utilisation de l'argon et à son fonctionnement à la pression atmosphérique. La plupart des éléments sont ionisés à plus de $90 \%$, seuls quelques éléments à haute énergie d'ionisation ne sont que partiellement ionisés $(\mathrm{H}, \mathrm{C}, \mathrm{N}, \mathrm{O}$, halogènes, $\mathrm{S}, \mathrm{Hg})$. Un autre avantage de ce système est le confinement de l'échantillon dans une zone centrale produite par effet de peau, grâce à l'utilisation d'un champ haute fréquence pour créer le plasma et donner de l'énergie aux ions. Les ions vont donc se trouver dans une zone de quelques millimètres de largeur, ce qui facilitera leur prélèvement.

\section{LA SPECTROMETRIE DE MASSE}

Le spectromètre de masse travaille habituellement à température ambiante et à très basse pression $(<10$ ${ }^{3} \mathrm{~Pa}$ ). Or, les ions sont créés dans le plasma à une température de l'ordre de $5000^{\circ}$ à $6000^{\circ} \mathrm{K}$ et à la pression atmosphérique. Une interface doit donc être réalisée entre le plasma et le spectromètre de masse 


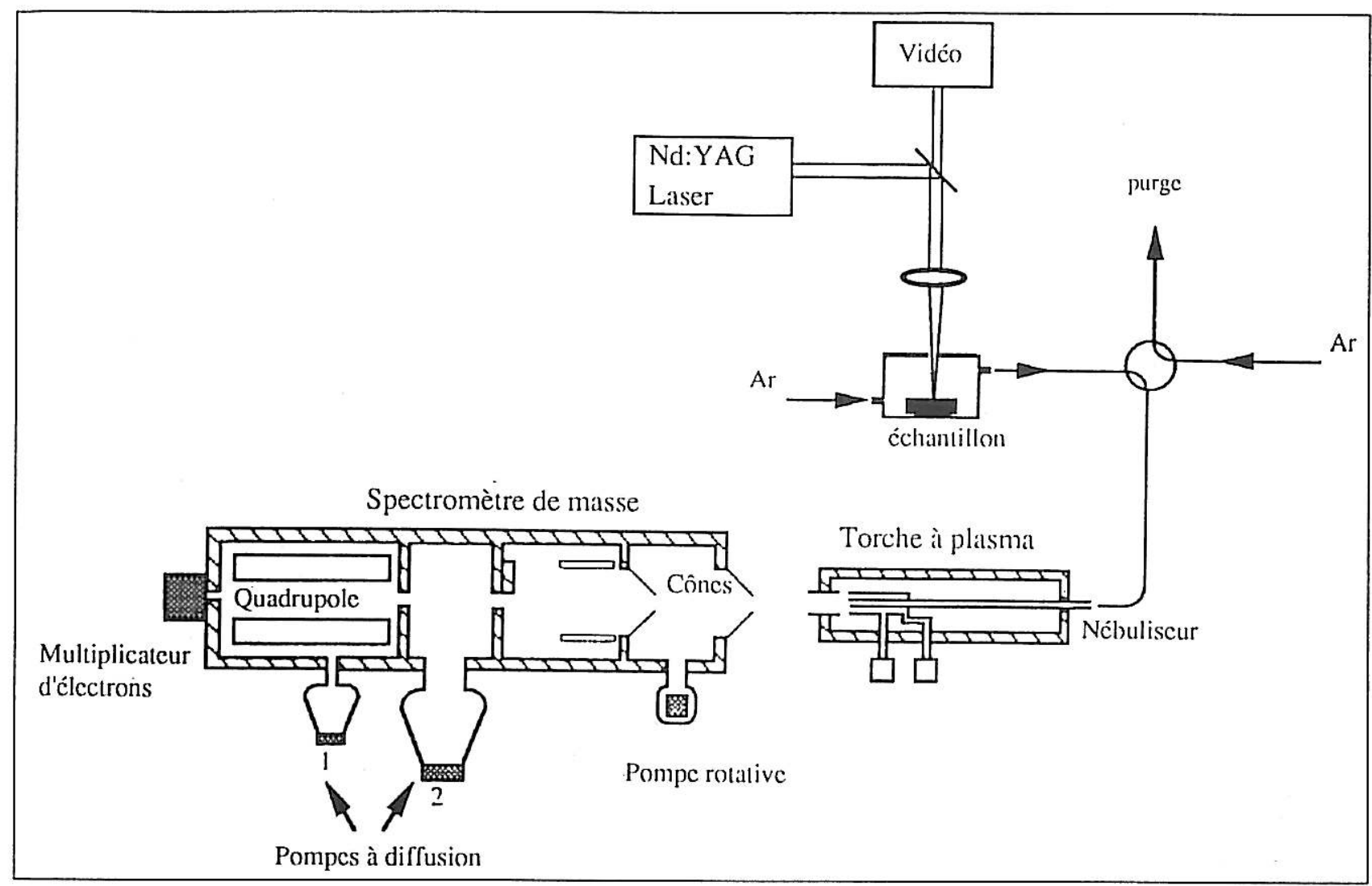

Fig. 1: Schéma de principe de l'appareil utilisé en LA-ICP-MS.

pour:

- passer de la pression atmosphérique à la pression de fonctionnement du spectromètre de masse;

- résister à une température élevée;

- extraire les ions en quantité suffisante.

Cette interface, qui utilise un système de pompage différentiel à trois étages et deux cônes, permet de prélever l'échantillon qui se trouve à la pression atmosphérique par la technique des jets supersoniques. A la sortie du second cône, le faisceau d'ions, fortement divergent, est refocalisé et rendu concentrique à l'axe du filtre quadripolaire par un système de lentilles électrostatiques. Le rapport signal/bruit est considérablement amélioré grâce à une lentille spécifique, placée dans l'axe des cônes, qui permet d'arrêter les photons émis par le plasma ("photon-stop»).

Le détecteur est un multiplicateur d'électrons de type «channeltron» qui permet d'obtenir une grande sensibilité pour de faibles teneurs. Deux modes de balayage peuvent être utilisés:

- «pic jumping», où l'on va sélectivement d'une masse à l'autre;

- «scanning», où l'on balaye l'ensemble des masses.

Les limites de détection sont sensiblement meilleures en mode "pic jumping".

L' appareil utilisé pour ce travail est un VG Plasma Quad 2+ EDR, le laser est un VG Laserlab A.

\section{RESULTATS}

\section{1 - CARACTERE NON DESTRUCTIF DE LA METHODE}

Les résultats obtenus, tant sur les objets métalliques que sur les verres, sont très prometteurs. Le diamètre des impacts est généralement inférieur à $50 \mu \mathrm{m}$ (pl. 1). Dans le cas des pièces de monnaie, l'analyse est effec- tuée sur la tranche de l'objet, le repérage visuel des impacts après l'analyse est impossible et reste très difficile avec un grossissement de 10 . Seule la marque noire due à un dépôt de carbone autour de l'impact peut renseigner sur l'endroit d'analyse. A noter que ce dépôt s'élimine par simple frottement avec les doigts. Dans le cas des verres, les mêmes observations s'imposent (pl. 2). Toutefois dans certains cas, un léger éclatement de la zone située autour de l'impact est observé. Cet éclatement localisé de l'échantillon est lié à la longueur d'onde du laser qui, située dans l'infra-rouge, se trouve dans la zone de transparence du verre. L'utilisation d'un laser travaillant dans l'ultra-violet (opacité du verre pour ces longueurs d'onde) devrait normalement permettre d'obtenir de meilleurs résultats. Cependant les impacts restent invisibles à l'oeil nu et leurs diamètres sont très inférieurs à ceux des trous occasionnés par la corrosion.

Les mesures de profil effectuées sur les impacts créés sur une plaque d'or nous montrent que les trous ont approximativement la forme d'un cône dont la profondeur est environ égale au dixième du diamètre. Pour un trou de $50 \mu \mathrm{m}$ de diamètre (un trou correspond à une série de 150 impacts laser), le volume prélevé est environ $3.300 \mu \mathrm{m}^{3}$ soit une masse comprise entre 30 et $70 \mathrm{ng}$ ( $1 \mathrm{ng}=10^{-9} \mathrm{~g}$ ) pour l'or et l'argent et de l'ordre de 8 à 10 ng pour le verre. Cinq dosages (cinq séries d'impacts) étant effectuées à différents endroits de l'objet par analyse, le prélèvement total est inférieur à $0,5 \mu \mathrm{g}$ (1 $\mu \mathrm{g}=10^{-6} \mathrm{~g}$ ) ce qui est 200 fois plus faible que la masse prélevée par A.A. Gordus (100 $\mu \mathrm{g}$ ) (Gordus, 1972) pour les analyses de monnaies d'or et d'argent par activation neutronique au réacteur, et de 2000 à 100000 fois plus faible que la masse généralement prélevée pour des analyses en solution par des méthodes classiques (entre 1 et $50 \mathrm{mg}$ au minimum: absorption atomique, colorimétrie, gravimétrie...). La méthode peut doncêtre considérée comme non destructive. 
Grossissement : X 25 par rapport à l'objet

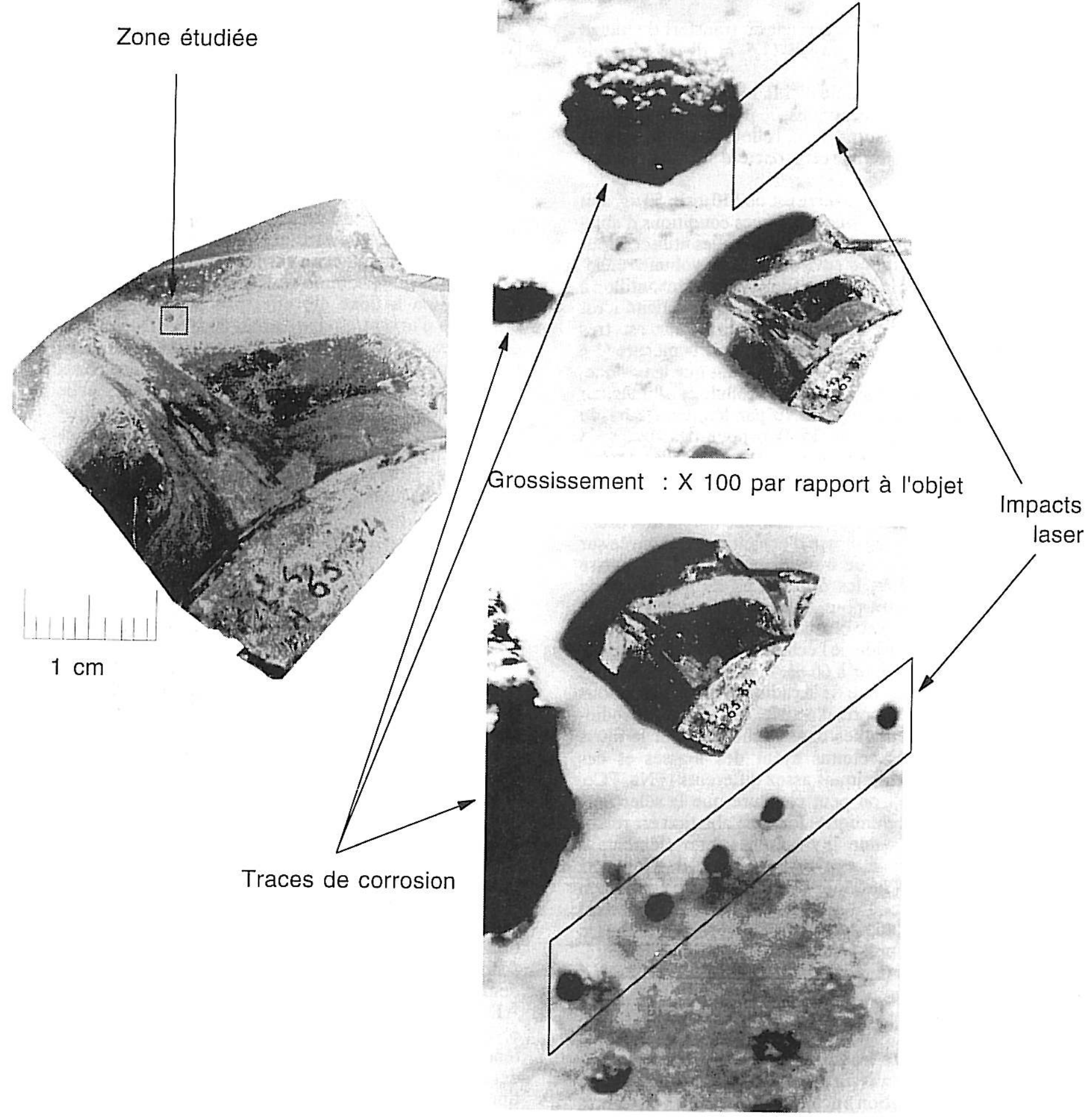




\section{2 - REPRODUCTIBILITE ET REPRESENTATIVI- TE DES RESULTATS}

Pour vérifier l'absence de sélectivité lors de l'ablation et mesurer le taux de transmission du nuage atomique entre la cellule d'ablation et la torche à plasma, un verre étalon (NIST 610 du National Bureau of Standard) préalablement irradié a été soumis à une ablation intensive (15 000 impacts à puissance élevée). Le nuage prélevé est recueilli sur un filtre positionné à la place de la torche. Des mesures de la radioactivité sur les différentes parties du dispositif expérimental ont été réalisées:

- le filtre;

- le tuyau de plastique servant au transfert du nuage entre la cellule et la torche $(1,5 \mathrm{~m}$ divisé en trois tronçons de $0,5 \mathrm{~m}$ chacun);

- le support de la cellule d'ablation.

Pour des raisons pratiques, il n'a pas été possible d'effectuer des mesures de la cellule. La radioactivité initiale et la masse du verre ont été mesurées avant l'expérience.

La perte de masse du verre est de $510 \mu \mathrm{g} \pm 50 \mu \mathrm{g}$, soit environ $0,034 \mu \mathrm{g}$ par impact dans ces conditions d'ablation intensive qui sont différentes de celles utilisées pour les analyses (à noter qu'environ $90 \%$ du volume réellement prélevé par le laser se redépose sur l'échantillon à proximité de l'impact). Sa perte de radioactivité n'est pas mesurable, car son ordre de grandeur est très inférieur à celui de l'erreur commise sur la mesure (1 à $5 \%$ ). Les mesures effectuées montrent que la radioactivité présente sur le support de la cellule et à l'intérieur des tuyaux n'est pas décelable par les détecteurs du Centre E. Babelon (Ge $15 \%$ ), pour des temps de mesure compris entre trois et quatre jours. Par contre, les mesures effectuées sur le filtre correspondent à une radioactivité moyenne égale à $0,027 \% \pm 0,003 \%$ de la radioactivité initiale du verre soit une masse de l'ordre de $340 \mu \mathrm{g} \pm 40 \mu \mathrm{g}$. La masse d'échantillon retrouvée sur le filtre est donc égale à $66 \% \pm 12 \%$ de la masse prélevée. Toutefois, les différences de géométrie de comptage observées pour le filtre et le verre font que cette valeur est un peu pessimiste et on peut penser que le taux de transmission de l'échantillon entre la cellule et la torche est supérieur à $66 \%$.

Une analyse plus fine de la radioactivité du filtre nous montre que les rapports d'activité des différents radioisotopes sont identiques dans l'échantillon et le filtre. Les radioisotopes retenus ayant des masses et des comportements chimiques assez différents $\left({ }^{22} \mathrm{Na},{ }^{57} \mathrm{Co}\right.$, $\left.{ }^{58} \mathrm{Co},{ }^{120} \mathrm{Sb},{ }^{139} \mathrm{Ce}\right)$, on peut conclure que la sélectivité entre les espèces chimiques lors de l'ablation est relativement faible, et que les rapports inter-éléments à l'intérieur du nuage sont représentatifs des rapports inter-éléments à l'intérieur de l'objet. Cette conclusion n'est valable que pour les objets dont la composition est homogène à l'échelle des $50 \mu \mathrm{m}$, il va de soit que dans le cas de matériaux présentant des phénomènes de ségrégation importants (cuivreux), ces résultats seraient remis en cause.

Afin de vérifier la validité et la représentativité des résultats obtenus par LA-ICP-MS, une comparaison a été effectuée entre les résultats obtenus par cette méthode et ceux obtenus sur les mêmes échantillons par les méthodes d'activation nucléaire développées au Centre E. Babelon pour ces matériaux.

Sept verres, trois obsidiennes et onze fragments de monnaies (sept d'or et quatre d'argent) ont été analysés par LA-ICP-MS. Cinquante-quatre éléments ont été systématiquement dosés dans les verres et l'obsidienne, et douze dans les monmaies, deux autres éléments faisant simplement l'objet d'un repérage. Il est à noter que d'un point de vue pratique, les problèmes posés par les monnaies et les verres sont différents. En effet, si l'étalonnage des mesures pour les verres ne pose pas de problème (utilisation des verres étalons internationaux: NIST610 et 612), nous ne disposons pas d'étalons certifiés pour l'analyse des monnaies d'or et d'argent. L'appareil a donc été étalonné non pas à partir d'échantillons de référence comme dans le cas des verres, mais à partir des pièces de monnaie préalablement analysées par activation protonique au Centre E. Babelon. Ceci explique que seuls des résultats qualitatifs aient été obtenus pour des éléments du groupe des platinoïdes comme l'iridium et le rhodium (éléments non dosés par PAA aux teneurs rencontrées dans les pièces). D'autre part, certains des éléments dosés dans les pièces étant présents à des teneurs proches des limites de détection de l'activation protonique, cela.implique un taux d'erreur relativement important sur les valeurs proposées et donc des difficultés à établir une courbe d'étalonnage précise.

Nous disposions de deux séries de fragments de pièces d'or, la première, très pure en or, et la seconde, relativement riche en argent et en cuivre. Une pièce de chaque série a été utilisée comme étalon. Pour les monnaies, la comparaison a donc été effectuée à partir de trois monnaies d'argent et cinq monnaies d'or. Dans le cas des verres, les résultats ont été comparés avec ceux obtenus par FNAA et pour l'un d'entre eux avec ceux obtenus par activation avec des protons de $12 \mathrm{MeV}$ et de $20 \mathrm{MeV}$ et par ICP-AES. Pour l'obsidienne, la comparaison a été effectuée d'une part avec les résultats obtenus par FNAA et d'autre part avec ceux publiés par cinq autres laboratoires français et étrangers pour des échantillons géologiques provenant des mêmes gisements.

\section{a) Le verre}

On observe un très bon accord entre les valeurs obtenues par LA-ICP-MS et par FNAA (fig. 2 et 3). Une étude détaillée des résultats montre que l'écart moyen observé entre les teneurs proposées par LA-ICP-MS et par FNAA est de l'ordre de $9 \%$ pour l'ensemble des éléments majeurs (aluminium, calcium, potassium, fer, magnésium, manganèse, sodium et silice) (tabl. 1).

Les verres "Sb plat 245" et «Sb plat" sont deux fragments provenant de la même pièce, les deux ont été analysés par FNAA, l'un d'entre eux l'a été par LA-ICPMS. Les verres «Sang3»et «V007» sont eux aussi identiques, tous deux ont été analysés par les deux méthodes.

Parmi les éléments majeurs, les écarts les plus importants sont observés pour le manganèse, il est à noter que le dosage de cet élément est fortement perturbé par le fer en FNAA. On observe d'ailleurs une nette corrélation entre les rapport $\mathrm{Mn} / \mathrm{Fe}$ des l'échantillon et les écarts obtenus pour le dosage du manganèse par les deux méthodes (fig. 4)

D'autre part, si on élimine des éléments comme le césium, le niobium, l'yttrium, l'argent et l'or dont les teneurs sont presques toujours égales aux limites de détection obtenues par FNAA, on note que, à l'exception de certains éléments pour lesquels des écarts importants sont systématiquement observés (chlore et cuivre), les écarts relatifs observés sont inférieurs à $10 \%$ dans $50 \%$ des cas et inférieurs à $20 \%$ dans $67 \%$ des cas. Les variations importantes observées pour le cuivre et le 


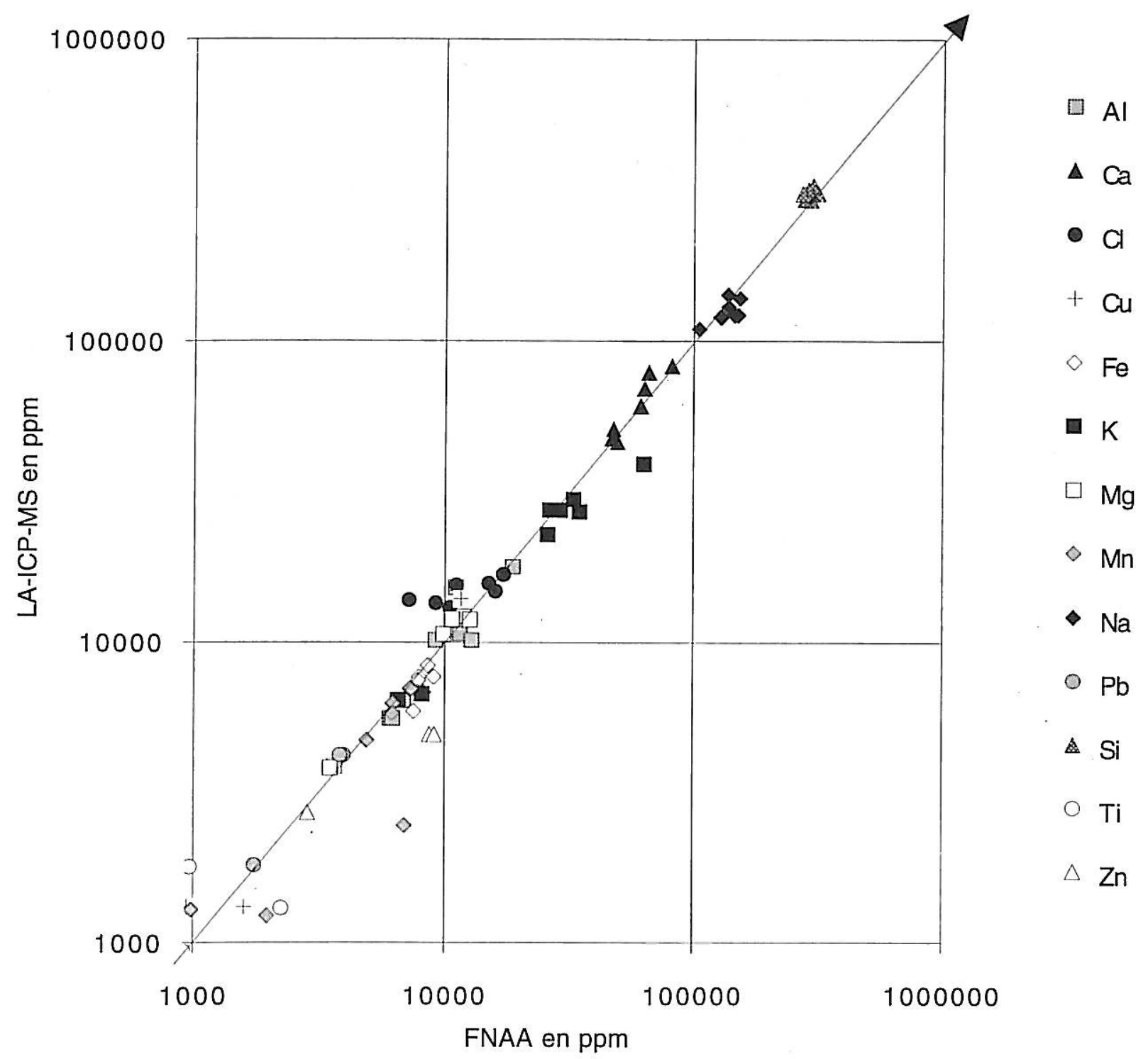

Fig. 2: Diagramme FNAA/LA-ICP-MS pour les éléments dont les teneurs sont supérieures à $1000 \mathrm{ppm}$ dans les verres.

chlore sont certainement dues d'une part aux difficultés importantes rencontrées pour doser le cuivre par FNAA dans les verres à fortes concentrations de cobalt et de nickel et d'autre part à une contamination de la surface des objets en chlore lors de la manipulation avec les doigts.

Si l'on étudie les écarts observés en fonction de l'ordre de grandeur des teneurs mesurées, on constate que la moyenne des variations observées est inférieure à $18 \%$ pour l'ensemble des teneurs supérieures à $100 \mathrm{ppm}$ et est de l'ordre de $27 \%$ pour l'ensemble des teneurs. Des disparités plus importantes sont observées sur certains éléments comme le zinc, le nickel, l'antimoine et l'arsenic. Ces résultats ne peuvent être interprétés comme étant dus à une hétérogénéité du matériau; en effet, chaque résultat obtenus par LA-ICP-MS est la moyenne de cinq mesures différentes, sur des points d'impacts différents, pour lesquels les écarts-types relatifs sont:

- inférieurs à $5 \%$ pour les éléments dont les teneurs sont supérieures à $1 \%$;

- compris entre 5 et $10 \%$ pour les éléments dont les teneurs sont comprises entre $10 \mathrm{ppm}$ et $1 \%$;

- de l'ordre de 10 à $20 \%$ pour les éléments présents à des teneurs inférieures à $10 \mathrm{ppm}$.

D'autre part, parmi les échantillons analysés par LAICP-MS se trouvaient deux échantillons identiques analysés sous des références différentes (Sang3 et V007); les variations relatives de teneurs observées entre les deux échantillons sont inférieures à $10 \%$ pour $88 \%$ des teneurs supérieures à $10 \mathrm{ppm}$ (fig. 5). Ces résultats permettent d'apprécier à la fois la reproductibilité de la méthode et l'homogénéité du matériau.

La comparaison entre les valeurs obtenues par PAA et ICP-AES d'une part et par LA-ICP-MS d'autre part donne des résultats comparables à ceux obtenus par comparaison avec la FNAA (tab. 2).

\section{b) L'obsidienne}

Le même bon accord général est observé pour l'obsidienne. Les écarts moyens sont toutefois plus importants que dans le cas des verres du fait des modes de comparaison adoptés. En effet deux types de comparaison ont été effectués:

- d'une part entre nos rapports élément/sodium (Gratuze et al., 1993) et ceux calculés à partir des valeurs données par LA-ICP-MS, ce qui implique l'addition des erreurs relatives liées au dosage de chaque élément.

- d'autre part entre les valeurs données par LA-ICPMS et celles publiées par cinq autres laboratoires français et étrangers pour des échantillons géologiques provenant des mêmes gisements (Cauvin et al., 1986; 
Francaviglia, 1990; MURR; Keller et Seifried, 1989; Renfrew et Cann, 1964). Dans ce cas, la comparaison est effectuée avec les teneurs moyennes, minimales et maximales données pour chaque gisement. D'une façon générale, les valeurs trouvées par LA-ICP-MS se situent dans les fourchettes des valeurs données pour les échantillons de mêmes origines (tab. 3 et fig. 6).

\section{c) L'or}

La comparaison effectuée à partir des résultats obtenus, par PAA et par LA-ICP-MS, sur les monnaies, met en évidence des écarts plus importants que ceux observés pour le verre (fig. 7). Les erreurs relatives pour chaque méthode oscillant entre 5 et $10 \%$ pour les éléments majeurs et mineurs et entre 5 et $15 \%$ pour les éléments traces, des variations comprises entre 20 et $30 \%$ sont explicables et ne peuvent pas, dans notre cas, être considérées comme reflétant une hétérogénéité du matériau ou un désaccord entre les deux méthodes. Cette dispersion importante des résultats est essentiellement imputable au mode d'étalonnage employé pour les analyses effectuées par LA-ICP-MS. On note toutefois un bon accord pour les éléments majeurs (or, argent et cuivre), la moyenne des écarts y est en effet inférieure à $20 \%$ pour les deux groupes de monnaies. Une disper- sion plus importante est observée pour les mineurs et les traces avec toutefois là encore de bons résultats pour des éléments comme le fer et le platine où l'écart relatif moyen reste inférieur à $30 \%$. Pour l'ensemble des éléments, la moyenne des écarts est inférieure à $38 \%$

\section{d) L'argent}

Pour l'argent, les réserves effectuées pour l'or s'appliquent aussi. On note toutefois un bien meilleur accord entre les valeurs obtenues par les deux méthodes pour les éléments mineurs et traces: la moyenne des écarts observés est en effet inférieure à $18 \%$ pour l'ensemble des éléments dosés (fig. 8). Les meilleurs résultats trouvés pour les pièces d'argent s'expliquent par les teneurs plus fortes rencontrées dans ces pièces pour les éléments mineurs et traces. Ceci a permis une plus grande précision lors des analyses par PAA et donc de bénéficier d'un étalon de meilleure qualité pour l'étalonnage en LA-ICP-MS.

\section{3 - LIMITES DE DETECTION}

Les limites de détection données sont calculées pour une acquisition en mode «pic jumping» sur un verre. $\mathrm{Si}$ l'on exclut quelques éléments qui nécessitent l'utilisa-

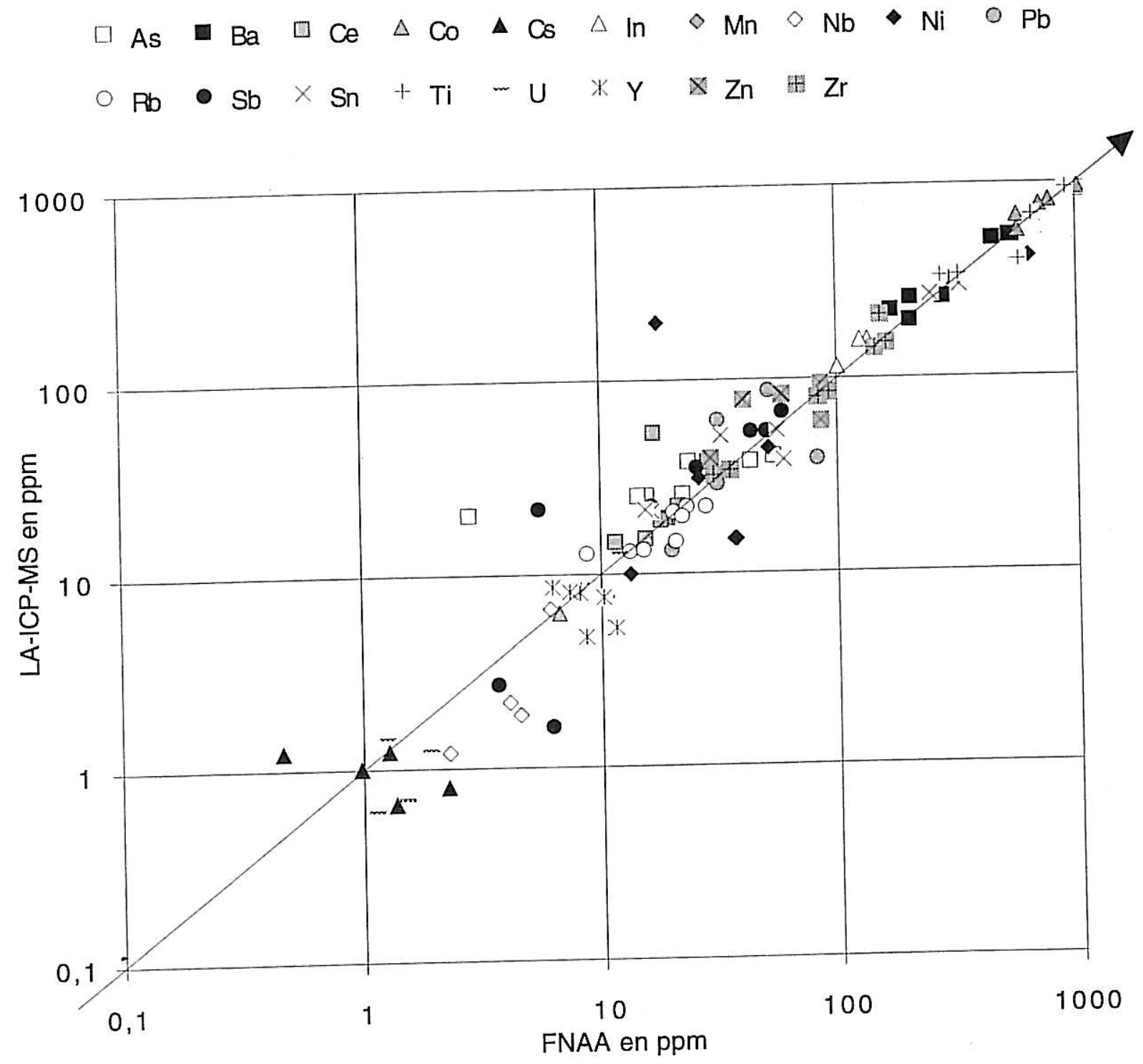

Fig. 3: Diagramme FNAA/LA-ICP-MS pour les éléments dont les teneurs sont inférieures à $1000 \mathrm{ppm}$ dans les verres. 


\begin{tabular}{|c|c|c|c|c|c|c|c|c|c|c|c|}
\hline & Verre & FNAA & L-I-M & $\Delta$ & $\Delta$ moyen & & Verre & FNAA & L-I-M & $\Delta$ & $\Delta$ moyen \\
\hline \multirow[t]{8}{*}{$\mathrm{Al}$} & Cadrix 9 & 1,87 & 1,78 & $4,9 \%$ & & \multirow[t]{8}{*}{$\mathrm{Mg}$} & Marrotte & 1,19 & 1,22 & $2,5 \%$ & \\
\hline & $A V 1 B$ & 1,62 & 1,52 & $6,3 \%$ & & & $A V 1 B$ & 1,12 & 1,07 & $4,5 \%$ & \\
\hline & Sb fil bleu & 0,68 & 0,64 & $6,4 \%$ & & & V007 & 0,36 & 0,39 & $5,6 \%$ & \\
\hline & V007 & 1,15 & 1,07 & $7,4 \%$ & & & Cadrix 9 & 1,26 & 1,19 & $5,8 \%$ & \\
\hline & Sb plat 245 & 0,61 & 0,56 & $7,9 \%$ & & & Sang 3 & 0,35 & 0,38 & $8,3 \%$ & \\
\hline & Sb plat & 0,62 & 0,56 & $10,1 \%$ & & & Sb plat & 1,07 & 1,19 & $10,6 \%$ & \\
\hline & Sang 3 & 0,92 & 1,02 & $10,4 \%$ & $7,6 \varepsilon$ & & Sb plat 245 & 1,06 & 1,19 & $10,9 \%$ & \\
\hline & Marrotte & 1,28 & 1,02 & $23,1 \%$ & $9,5 \%$ & & SB fil bleu & 1,07 & 1,19 & $11,0 \%$ & $7,4 \%$ \\
\hline \multirow[t]{8}{*}{$\mathrm{Ca}$} & Sb plat 245 & 6,13 & 6,09 & $0,6 \%$ & & \multirow[t]{8}{*}{$\mathrm{Mn}$} & Cadrix 9 & 0,62 & 0,63 & $0,7 \%$ & \\
\hline & V007 & 8,18 & 8,26 & $1,1 \%$ & & & AV $1 B$ & 0,24 & 0,25 & $1,6 \%$ & \\
\hline & Sb plat & 6,16 & 6,09 & $1,2 \%$ & & & Sb plat 245 & 0,73 & 0,70 & $3,6 \%$ & \\
\hline & Marrotte & 4,67 & 4,77 & $2,0 \%$ & & & Marrotte & 0,49 & 0,47 & $3,6 \%$ & \\
\hline & AV 1B & 6,66 & 6,99 & $4,8 \%$ & & & Sb plat & 0,73 & 0,70 & $3,7 \%$ & \\
\hline & Sb fil bleu & 4,93 & 4,64 & $6,3 \%$ & & & Sb fil bleu & 0,67 & 0,58 & $5,9 \%$ & $3,2 \%$ \\
\hline & Cadrix 9 & 4,76 & 5,12 & $7,2 \%$ & $3,3 \%$ & & V007 & 0,98 & 0,13 & $26,8 \%$ & \\
\hline & Sang 3 & 6,60 & 7,89 & $17,8 \%$ & $5,1 \%$ & & Sang 3 & 0,20 & 0,12 & $46,6 \%$ & $11,6 \%$ \\
\hline \multirow[t]{8}{*}{$\mathrm{Fe}$} & Cadrix 9 & 0,85 & 0,84 & $2,1 \%$ & & \multirow[t]{8}{*}{$\mathrm{Na}$} & Marrotte & 13,8 & 14,2 & $3,1 \%$ & \\
\hline & Sang 3 & 0,79 & 0,77 . & $3,0 \%$ & & & AV $1 B$ & 11,4 & 11,0 & $3,2 \%$ & \\
\hline & Marrotte & 0,78 & 0,75 & $4,7 \%$ & & & Cadrix 9 & 13,8 & 113,0 & $6,1 \%$ & \\
\hline & AV 4087 & 0,45 & 0,42 & $6,3 \%$ & $4,0 \%$ & & V007 & 12,8 & 12,0 & $6,2 \%$ & \\
\hline & Sb plat 245 & 0,78 & 0,68 & $13,1 \%$ & & & Sang 3 & 12,9 & 12,0 & $7,1 \%$ & \\
\hline & V007 & 0,91 & 0,77 & $16,4 \%$ & & & Sb fil bleu & 15,3 & 13,8 & $10,1 \%$ & $6,0 \%$ \\
\hline & Sb plat & 0,82 & 0,68 & $18,5 \%$ & $9,2 \%$ & & Sb plat 245 & 14,6 & 12,2 & $17,9 \%$ & \\
\hline & Sb fil bleu & 0,75 & 0,59 & $24,0 \%$ & $11,0 \%$ & & Sb plat & 15,0 & 12,2 & $20,8 \%$ & $9,3 \%$ \\
\hline \multirow[t]{8}{*}{$\mathrm{K}$} & V007 & 0,65 & 0,64 & $1,1 \%$ & & \multirow[t]{8}{*}{$\mathrm{Si}$} & Marrotte & 29,5 & 29,3 & $0,7 \%$ & \\
\hline & Sb plat 245 & 2,64 & 2,75 & $4,0 \%$ & & & Sang 3 & 31,6 & 30,9 & $2,1 \%$ & \\
\hline & Sb plat & 2,90 & 2,75 & $5,3 \%$ & & & Cadrix 9 & 27,9 & 29,5 & $5,6 \%$ & \\
\hline & Marrotte & 3,28 & 2,99 & $9,5 \%$ & & & $A V 1 B$ & 29,1 & 30,8 & $5,7 \%$ & \\
\hline & Sb fil bleu & 2,58 & 2,28 & $12,0 \%$ & $6,4 \%$ & & V007 & 30,1 & 32,8 & $8,5 \%$ & \\
\hline & $A V 1 B$ & 4,53 & 3,92 & $14,6 \%$ & & & Sb fil bleu & 28,9 & 31,8 & $9,5 \%$ & \\
\hline & Sang 3 & 0,81 & 0,67 & $18,7 \%$ & $9,3 \%$ & & Sb plat 245 & 28,0 & 30,7 & $9,5 \%$ & \\
\hline & cadrix 9 & 3,47 & 2,71 & $24,7 \%$ & $11,2 \%$ & & Sb plat & $27+4$ & 30,7 & $11,5 \%$ & $6,6 \%$ \\
\hline & & & & & & & Moyenne & des & écarts & $9,0 \%$ & \\
\hline
\end{tabular}

Tab. 1: Comparaison des valeurs obtenues par FNAA et LA-ICP-MS pour les éléments majeurs pour les sept verres étudiés (concentration en \%).

tion 'd'isotopes moins sensibles mais libres d'interférences isobariques, les valeurs obtenues sont très bonnes. La majorité des limites de détection est inférieure à la partie par million, elles décroissent avec le numéro atomique de l'élément, et sont à quelques exceptions près comprises entre 0,01 et 0,3 ppm à partir du rubidium.

D'une façon générale les limites de détection obte-. nues par LA-ICP-MS sont 10 à 100 fois inférieures à celles obtenues par nos méthodes d'activation: entre 0,5 et 50 à $100 \mathrm{ppm}$ en mode non destructif pour la FNAA et le PAA pour les mineurs et traces (fig. 9). Si l'on compare les valeurs obtenues par ablation à celles généralement publiées dans la littérature pour l'ICPMS en solution: 0,1 à $0,01 \mathrm{ng} / \mathrm{l}$, on s'aperçoit que les limites obtenues par ablation sont plus élevées que celles obtenues en solution même si l'on tient compte d'un facteur de dilution de 500 (soit pour l'ICP-MS en solution des limites comprises entre 0,05 et $0,005 \mathrm{ppm}$ sur le solide). Ce gain en sensibilité obtenu en travaillant en solution est cependant limité par certains inconvénients:

- apport d'impuretés par les acides

- volatilisation de certains éléments lors de la mise en solution
- interférences isobariques liées à la formation d'espèces moléculaires entre les atomes de l'échantillon et ceux du solvant (eau, acides), ou entre l'argon et le solvant.

De plus, dans notre cas, ceci signifie un prélèvement plus important, ce qui rend la méthode destructive.

\section{4 - ELEMENTS DOSES ET PROBLEMATIQUES ARCHEOLOGIQUES}

\section{a) Le verre et l'obsidienne}

Dans les verres, l'activation neutronique nous permet d'analyser une trentaine d'éléments (tab. 4) avec des limites de détection comprises entre 1 et $50 \mathrm{ppm}$ pour les éléments traces et de l'ordre de 0,1 à $0,01 \%$ pour les éléments majeurs et mineurs. La problématique archéologique étudiée concerne l'origine des matières colorantes utilisées par les verriers et plus précisement le cobalt (Gratuze, Soulier et al., 1992). Il est donc particulièrement intéressant de doser les éléments associés au cobalt dans la nature; ces éléments, qui varient selon les mines étudiées, sont principalement le nickel et l'arsenic mais aussi le zinc, le plomb, l'indium, l'argent, l'or, le cadmium, le bismuth et le molybdène. 


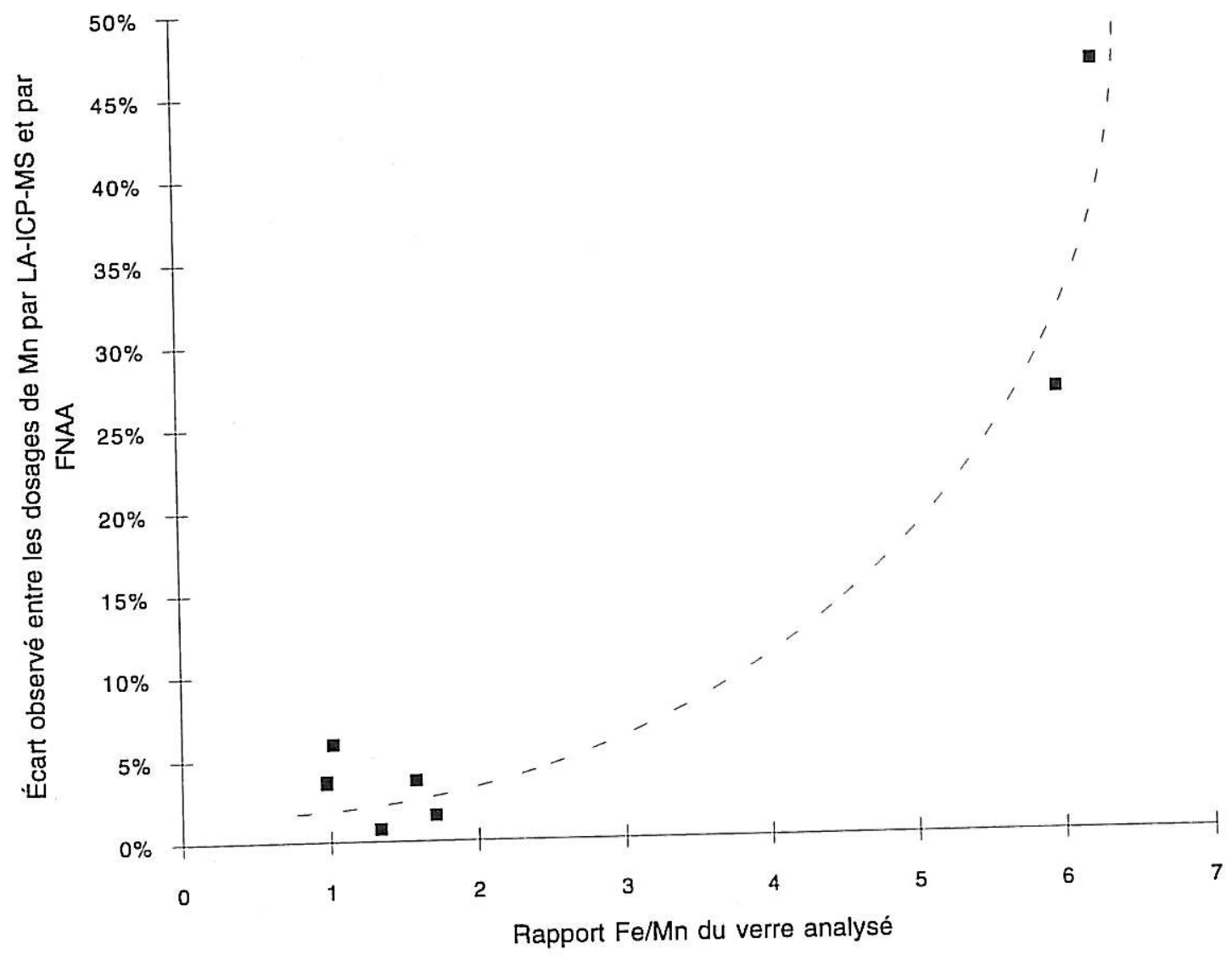

Fig. 4: Ecart observé entre les deux méthodes pour le dosage du manganèse en fonction du rapport des teneurs Fe/Mn de l'échantillon.

\begin{tabular}{|c|c|c|c|c|c|}
\hline élément $/$ méthode & FNAA & PAA 12 MeV & PAA 20 MeV & ICP-AES & LA-ICP-MS \\
\hline$\% \mathrm{Si}$ & 27,9 & & & & 29,5 \\
$\% \mathrm{Al}$ & 1,87 & & & 1,79 & 1,78 \\
$\% \mathrm{Na}$ & 13,8 & & 12,2 & 12,8 & 13,0 \\
$\% \mathrm{~K}$ & 3,47 & & & 2,54 & 2,71 \\
$\% \mathrm{Mg}$ & 1,26 & & & 1,1 & 1,19 \\
$\% \mathrm{Ca}$ & 4,76 & 4,98 & 4,37 & 4,35 & 5,12 \\
$\% \mathrm{Fe}$ & 0,85 & 0,88 & 0,79 & 0,78 & 0,84 \\
$\% \mathrm{Ti}$ & 0,22 & 0,24 & 0,29 & 0,22 & 0,13 \\
$\% \mathrm{Mn}$ & 0,62 & & 0,56 & 0,59 & 0,63 \\
$\mathrm{As}$ & 2,8 & 39,3 & 2,4 & & 20,5 \\
$\mathrm{Ba}$ & 452,8 & & 474,8 & & 515,2 \\
$\mathrm{Ce}$ & 28,1 & & 22,9 & & 37,8 \\
$\mathrm{Cl}$ & 11105 & & & & 15443 \\
$\mathrm{Co}$ & 6,6 & & 16,8 & 18,2 & 6,3 \\
$\mathrm{Cu}$ & 11651 & 13470 & 13408 & 14500 & 13931 \\
$\mathrm{Nb}$ & 6,9 & & & & 6,6 \\
$\mathrm{Ni}$ & 13,3 & & 13,2 & & 10 \\
$\mathrm{~Pb}$ & 31,2 & & 67,6 & 88,5 & 61,7 \\
$\mathrm{Rb}$ & 8,5 & & 14,7 & & 12,8 \\
$\mathrm{Sb}$ & 25,4 & 29,4 & 45,9 & 56 & 35,2 \\
$\mathrm{Sn}$ & 32,3 & & 30,6 & & 51,1 \\
$\mathrm{Sr}$ & & 297,5 & 242,7 & & 270 \\
$\mathrm{~V}$ & & 23,8 & 46,9 & & 20 \\
$\mathrm{Y}$ & 8,1 & 12,1 & 10,8 & & 8,0 \\
$\mathrm{Zn}$ & 58,4 & 95,6 & 66,4 & & 83 \\
$\mathrm{Zr}$ & 144,6 & 162,1 & 176 & & 142,3 \\
\hline
\end{tabular}

Tab. 2: Comparaison des résultats obtenus par cinq méthodes différentes pour le verre cadrix 9 (majeurs en \%, autres éléments en ppm). 


\begin{tabular}{|c|c|c|c|c|}
\hline Elément & Publié par & $\begin{array}{c}\text { Valeur } \\
\text { moyenne }\end{array}$ & $\begin{array}{c}\text { Valeur } \\
\text { minimale }\end{array}$ & $\begin{array}{c}\text { Valeur } \\
\text { maximale }\end{array}$ \\
\hline $\mathrm{Ba}$ & $\begin{array}{c}\text { Cauvin } \\
\text { Keller } \\
\text { LA-ICP-MS } \\
\end{array}$ & $\begin{array}{c}2,25 \\
2 \\
3,8 \\
\end{array}$ & 0,5 & 4 \\
\hline $\mathrm{Eu}$ & $\begin{array}{c}\text { Cauvin } \\
\text { MURR } \\
\text { LA-ICP-MS } \\
\end{array}$ & $\begin{array}{l}0,55 \\
0,70 \\
0,56 \\
\end{array}$ & 0,1 & 1 \\
\hline $\mathrm{Nd}$ & $\begin{array}{c}\text { Francaviglia } \\
\text { MURR } \\
\text { LA-ICP-MS }\end{array}$ & $\begin{array}{l}102 \\
74,4 \\
97,7 \\
\end{array}$ & 95 & 110 \\
\hline $\mathrm{Nb}$ & $\begin{array}{c}\text { Francaviglia } \\
\text { Cauvin } \\
\text { Keller } \\
\text { LA-ICP-MS } \\
\end{array}$ & $\begin{array}{c}65 \\
50 \\
61 \\
60,7 \\
\end{array}$ & $\begin{array}{l}61 \\
42\end{array}$ & $\begin{array}{l}72 \\
58\end{array}$ \\
\hline $\mathrm{Rb}$ & $\begin{array}{c}\text { Francaviglia } \\
\text { Keller } \\
\text { MURR } \\
\text { LA-ICP-MS } \\
\end{array}$ & $\begin{array}{l}248 \\
229 \\
225 \\
248 \\
\end{array}$ & 242 & 263 \\
\hline $\mathrm{Zr}$ & $\begin{array}{c}\text { Cauvin } \\
\text { Francaviglia } \\
\text { Keller } \\
\text { MURR } \\
\text { LA-ICP-MS }\end{array}$ & $\begin{array}{l}1185 \\
1168 \\
1273 \\
1210 \\
1258 \\
\end{array}$ & $\begin{array}{l}1063 \\
1138\end{array}$ & $\begin{array}{l}1308 \\
1261\end{array}$ \\
\hline
\end{tabular}

Tab. 3: Comparaison des résultats obtenus par LA-ICP-MS avec ceux publiés par d'autres laboratoires pour les obsidiennes d'un des gisements de la région de Bingol (Turquic), valeurs en ppm.

Parmi ces dix éléments, seuls les cinq premiers sont dosés en routine par activation, l'or et l'argent le sont occasionnellement et les autres ne le sont pas. Tous ces éléments sont par contre dosés sans aucun problème par LA-ICP-MS avec des limites de détection de 10 à 100 fois inférieures aux nôtres.

Parmi les verres étudiés par LA-ICP-MS, la mise en évidence de fortes concentrations de bismuth et la présence de molybdène à l'intérieur de l'un d'eux nous a permis de confirmer sans aucun doute l'origine supposée du colorant utilisé. De même, le dosage de l'indium à des concentrations inférieures aux limites de détection de la FNAA sur deux autres échantillons a permis d'envisager leur regroupement avec d'autres verres. Le dosage de l'ensemble des métaux de la première série de transition (chrome, manganèse, fer, cobalt, nickel et

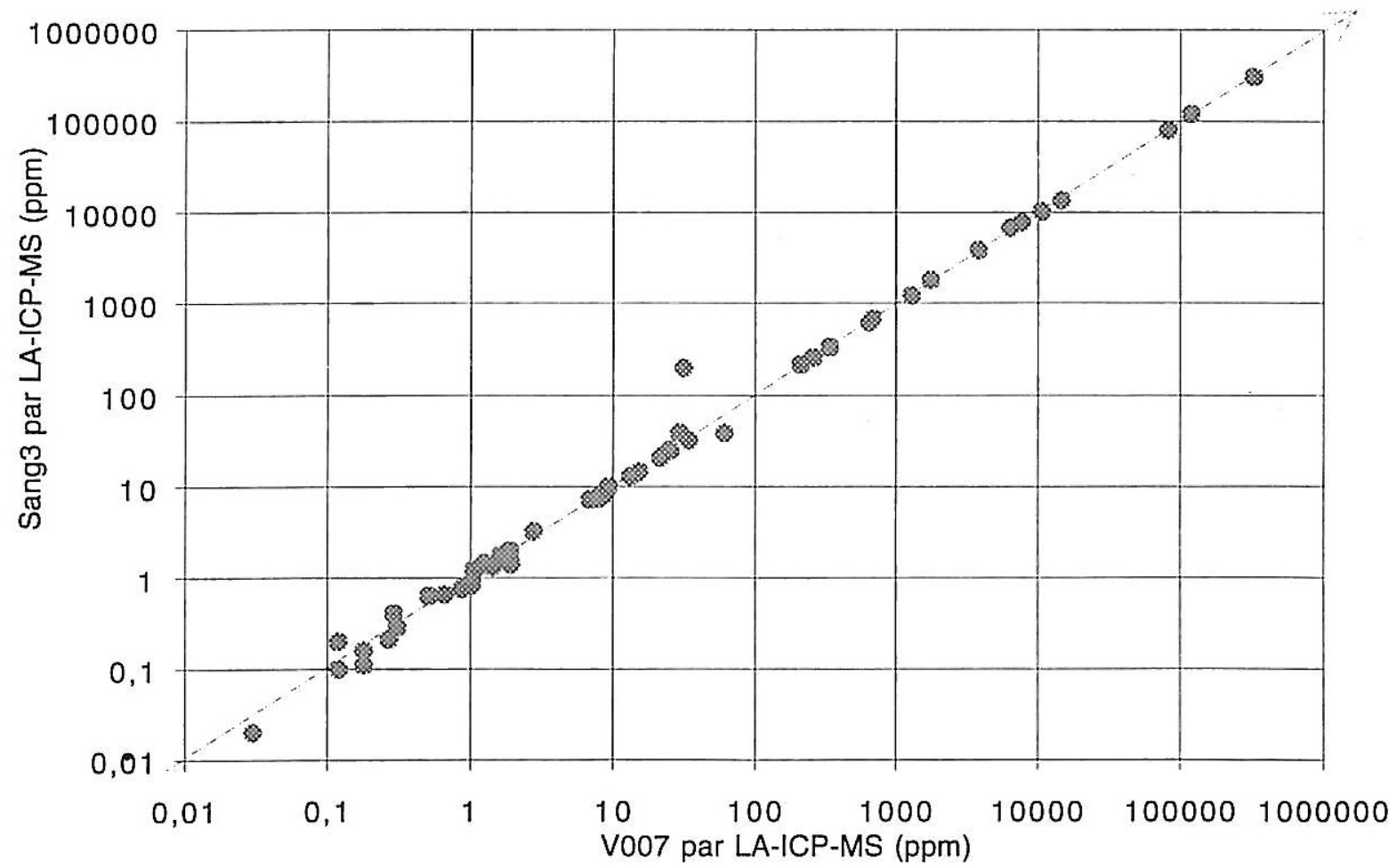

Fig. 5: Comparaison des résultats obtenus sur deux échantillons de verre identiques par LA-ICP-MS. 


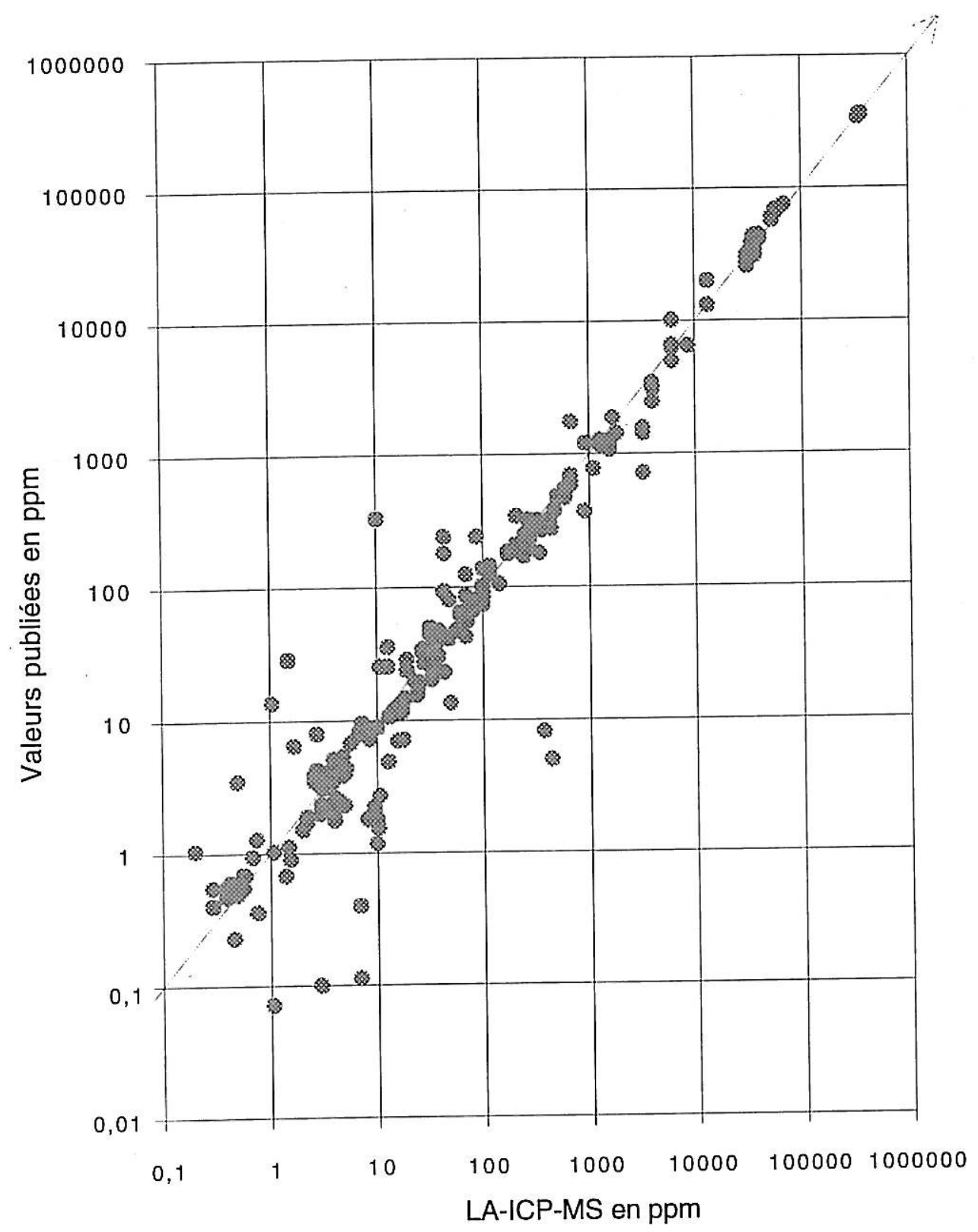

Fig. 6: Comparaison entre les teneurs moyennes publiées pour trois gisements d'obsidienne par cinq auteurs et celles obtenues par LA-ICP-MS pour des échantillons de même provenance.

cuivre) est particulièrement intéressant pour l'étude des colorants du verre: le cuivre par exemple qui a un rôle important dans la coloration des verres est difficile à doser par FNAA dans les verres à fortes concentrations en cobalt et en nickel. L'étude des premiers résultats obtenus sur le dosage des terres rares (non décelable par FNAA) semble indiquer que leur utilisation pour identifier les sites de production est envisageable. Le nombre d'analyses effectuées ne permet toutefois pas encore de disposer d'une statistique suffisante.

L'analyse non destructive des outils en obsidienne a été développée au Centre E. Babelon dans une optique différente de celle du verre. Dans ce matériau, les éléments majeurs interviennent moins que les éléments traces pour déterminer les provenances. Il nous a alors semblé intéressant de modifier le mode opératoire développé pour l'analyse du verre afin de permettre une plus grande cadence d'analyses tout en conservant à notre méthode un pouvoir de discrimination des gisements d'obsidienne comparable à celui obtenu par le dosage des terres rares. Les modifications apportées nous ont conduits à présenter nos résultats sous la forme de rapports entre les éléments dosés et le sodium. Ceci crée parfois certaines difficultés pour comparer nos valeurs avec celles publiées par certains auteurs qui ne dosent pas le sodium. Toutefois, si les éléments les plus utilisés pour différentier les sources d'obsidienne sont les terres rares, les méthodes utilisées pour leurs dosages (essentiellement l'activation neutronique au réacteur) ont l'inconvénient d'être destructives pour l'objet.

L'intérêt de la méthode LA-ICP-MS apparaît ici pleinement. Elle permet le dosage non destructif d'un nombre important d'éléments (tab. 5) dont les terres rares avec des limites de détections comparables, voir meilleures, à celles obtenues par activation neutronique. De plus, les cadences d'analyses sont très supérieures à celles des méthodes développées au Centre E. Babelon (plusieurs échantillons par heure par rapport à une dizaine d'échantillons par semaine) ce qui permet de répondre aux demandes importantes formulées par les préhistoriens dans ce domaine. 

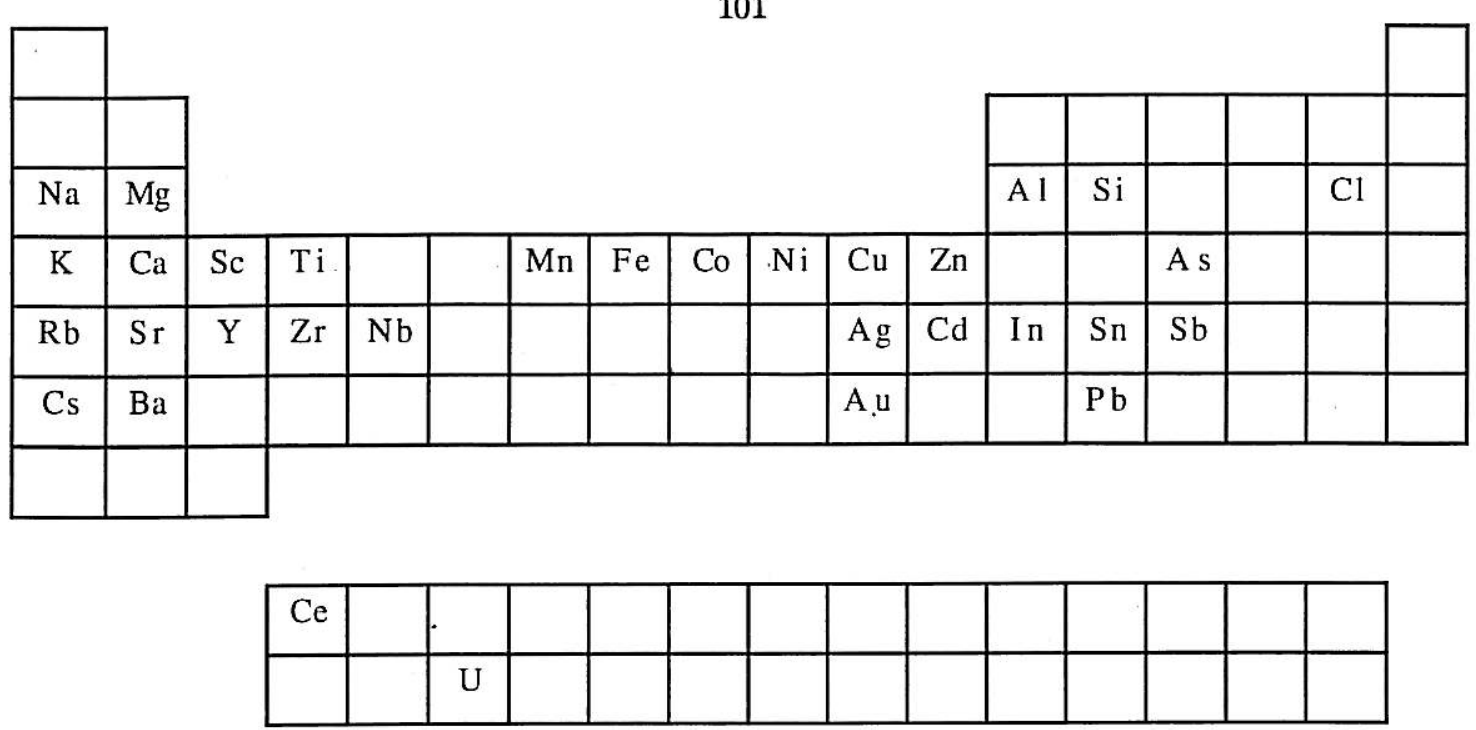

Tab. 4: Eléments dosés par activation neutronique dans les verres et l'obsidienne.

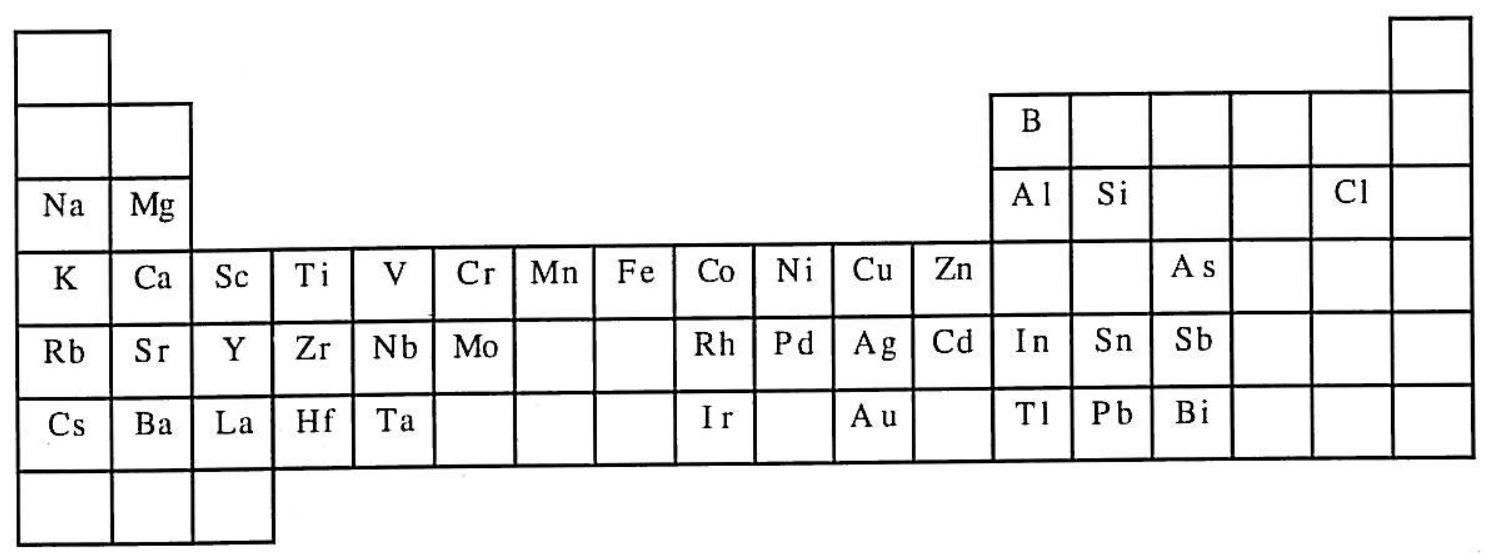

\begin{tabular}{|c|c|c|c|c|c|c|c|c|c|c|c|c|c|}
\hline Ce & Pr & Nd & & Sm & Eu & Gd & Tb & Dy & Ho & Er & Tm & Yb & Lu \\
\hline Th & & U & & & & & & & & & & & \\
\hline
\end{tabular}

Tab. 5: Eléments dosés par LA-ICP-MS dans les verres et les métaux.

\section{b) Les monnaies d'or et d'argent}

L'étude du monnayage d'or et d'argent a différents objectifs:

- l'étude des variations et des modes d'altérations du titre des monnaies;

- l'étude des refontes;

- l'étude des provenances des métaux utilisés.

Si les méthodes développées au Centre E. Babelon permettent pleinement de répondre au premier objectif et partiellement au second (tab. 6), l'étude des provenances des métaux se heurte par contre à de nombreuses difficultés que peu de laboratoires parviennent à résoudre. L'étude des platinoïdes pour l'or et l'argent ou la détermination des rapports isotopiques du plomb dans le cas de l'argent permettent cependant dans certains cas l'obtention de résultats intéressants.

Au Centre E. Babelon, l'etude de certaines problématiques archéologiques liées à ces problèmes de provenance a nécessité la mise au point de méthodes spéciales: la thermalisation des neutrons de cyclotron pour doser de façon non-destructive, les traces d'indium dans une matrice d'argent et l'étude de l'arrivée de l'argent du Potosi en Europe à partir du XVIème siècle (Guerra et Barrandon, 1988). Une étude identique menée sur l'or du Brésil (Barrandon et al., à paraître) a pu être effectuée uniquement grâce au dosage du palladium, élément bien dosé par activation protonique (limite de détection de l'ordre de $0,5 \mathrm{ppm}$ ).

Les possibilités offertes par l'ICP-MS pour le dosage de tous les platinoïdes et d'autres éléments traces avec une très bonne sensibilité, nous permettent d'envisager l'application de cette méthode au traçage des minerais d'or et d'argent et à l'étude des refontes. La précision des résultats obtenus actuellement sur les concentrations des différents isotopes du plomb limite par contre sérieusement les possibilités d'appliquer cette technique à la détermination des rapports isotopiques. Toutefois, les recherches effectuées par des équipes de géologues pour déterminer avec une précision suffisante ces rapports par LA-ICP-MS laissent entrevoir la possibilité d'utiliser cette technique pour identifier les mines de plomb argentifère exploitées pour la frappe des monnaies.

\section{CONCLUSION}

Les premiers résultats obtenus au cours de cette étude, démontrent les potentialités de caractérisation non destructive de certains archéomatériaux, offertes par l'association de l'ablation laser avec la spectrométrie de masse couplée aux plasmas induits par hautes 


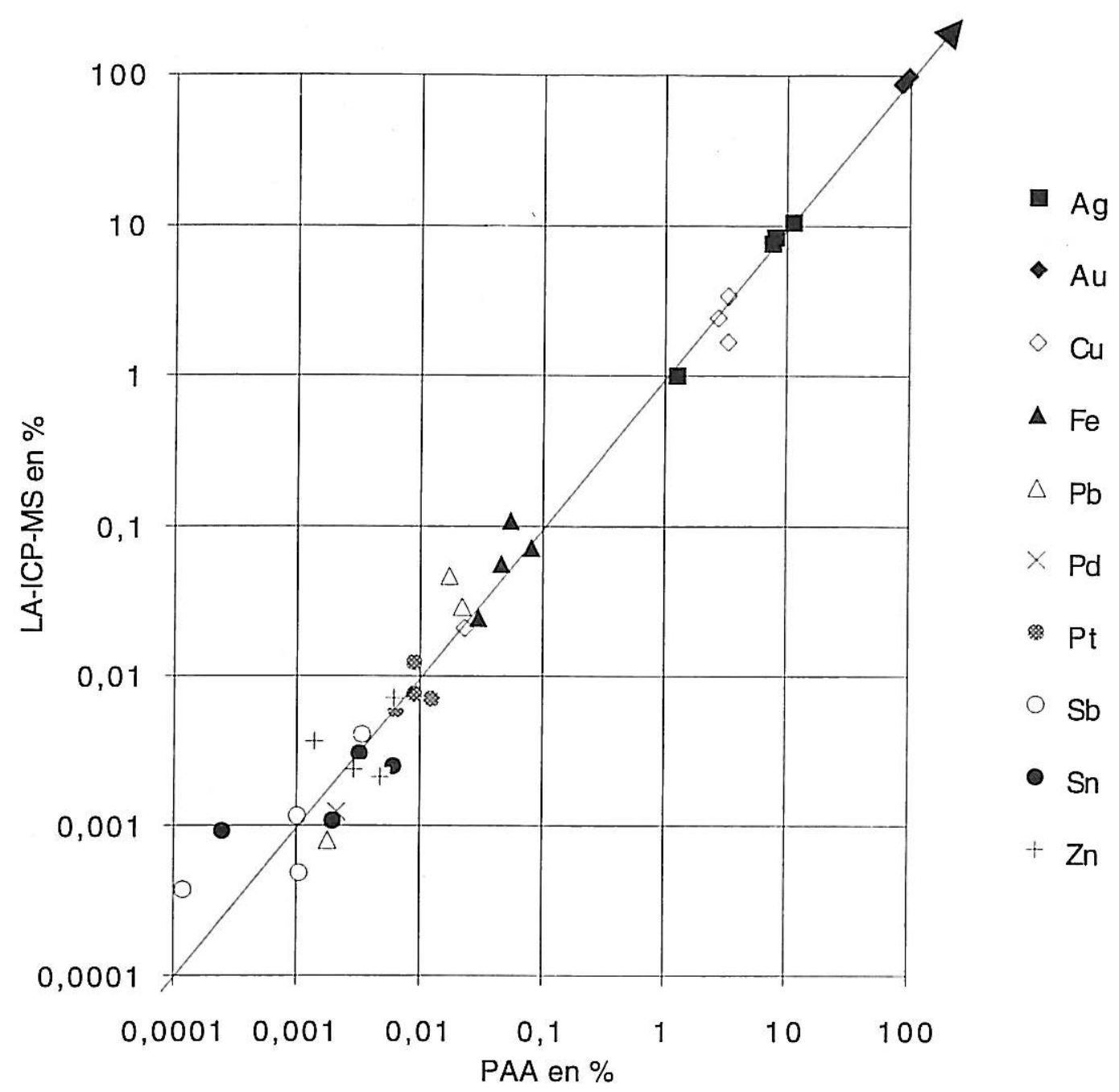

Fig. 7: Comparaison des résultats obtenus par PAA et par LA-ICP-MS pour les pièces d'or.

fréquences. Bien que les domaines d'application étudiés pour l'instant soit restreints, on peut cependant d'ores et déjà exclure l'application de cette méthode à l'étude non destructive de matériaux hétërogènes ou affectés par des phénomènes de corrosion importants tels que les cuivreux ou les ferreux. Cette méthode doit donc avant tout être considérée comme une méthode complémentaire des méthodes non destructives développées par le Centre E. Babelon. même si, dans le cas des matériaux étudiés au cours de ce travail (or, argent, verres synthétiques et naturels), elle peut être envisagée comme une méthode de substitution. Là encore toutefois les méthodes nucléaires conservent leur intérêt comme méthodes de contrôle et comme méthodes d'étalonnage. On notera cependant que dans le cas d'objets trop corrodés en verres, obsidienne ou même en argent, la méthode perd un peu de son intérêt non-destructif car une pré-ablation devra être effectuée avant l'analyse afin de dégager un partie saine de l'objet.

Les analyses par LA-ICP-MS peuvent par contre jouer un rôle très important pour l'étude des objets composites:

- verres décorés avec des filets de verres colorés ou peints,

- bijoux émaillés, sertis de pierres ou composés de plusieurs alliages,

- soudures présentes sur certains objets.

Les résultats obtenus au cours de ce travail sur un pied de coupe en verre bleu, décoré de motifs blancs et rouges ont permis de caractériser non destructivement le verre et son décor.

Ce type d'information ne peut être obtenu ni par les méthodes globales comme l'activation neutronique ni par des méthodes plus ponctuelles comme l'activation protonique (nombre d'éléments détectés trop faible et profondeur analysée trop importante) développées par le Centre E. Babelon. Seules des méthodes comme le PIXE ou la micro-fluorescence $X$ permettent à l'heure actuelle d'effectuer dans certain cas de tels analyses, mais là encore l'utilisation de ces méthodes est souvent limitée par les géométries complexes de ce type d'objets.

Parmi les autres champs d'application de cette méthode, se trouve la caractérisation des inclusions des céramiques. Nous quittons ici le domaine des analyses non destructives, étant donnée que ce type d'étude, actuellement en cours d'essai, est effectué à partir de lames minces utilisées pour l'analyse pétrographique en lumière polarisée. Les possibilités d'analyse à partir de solutions ne doivent pas non plus être négligées lorsque l'échantillon ne présente pas d'intérêt historique particulier. L'ICP-MS peut être alors une méthode performante et rapide pour l'analyses des scories et des minerais et par là même permettre l'étude des processus de métallurgie antique et du commerce de certain métaux. L'utilisation de l'association: ablation laser et spectro- 

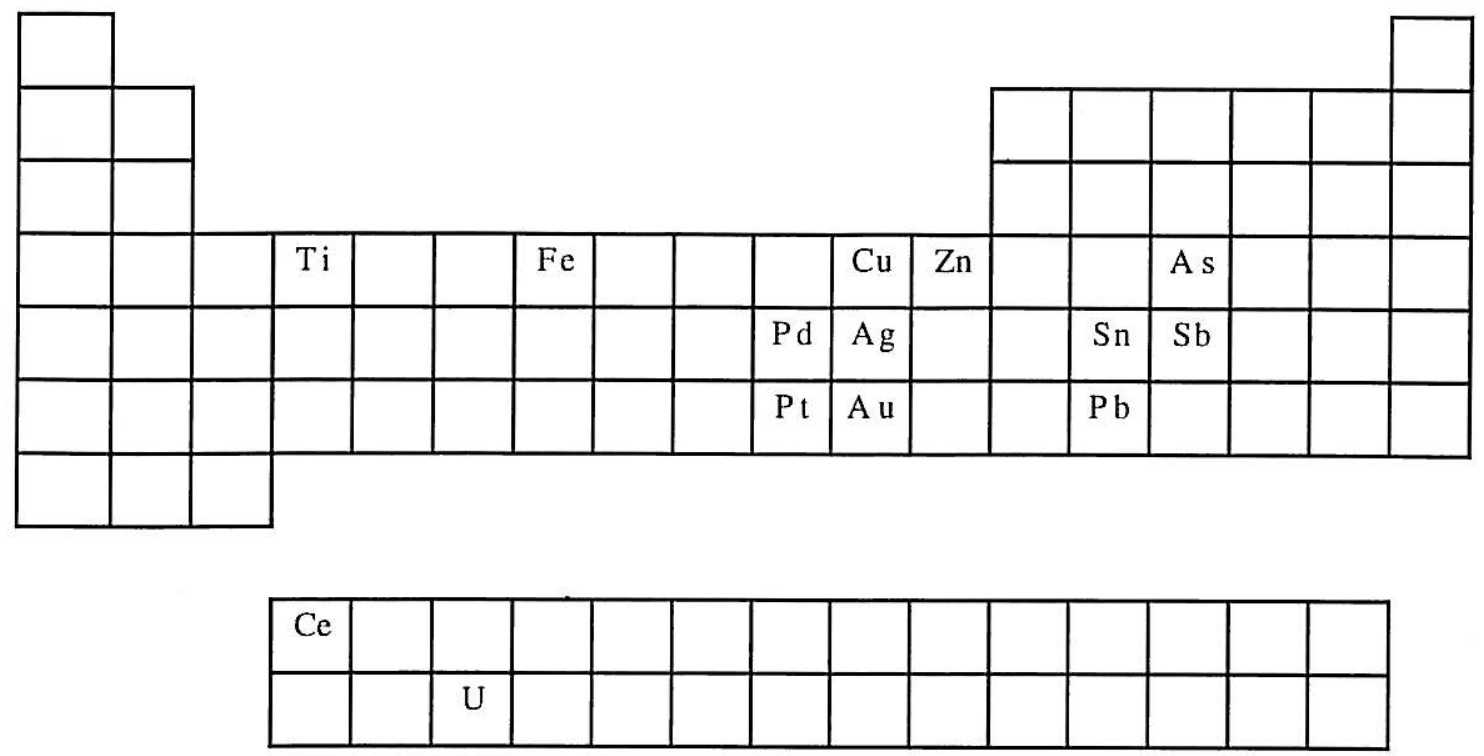

Tab. 6: Eléments dosés en routine par activation protonique dans l'or et l'argent.

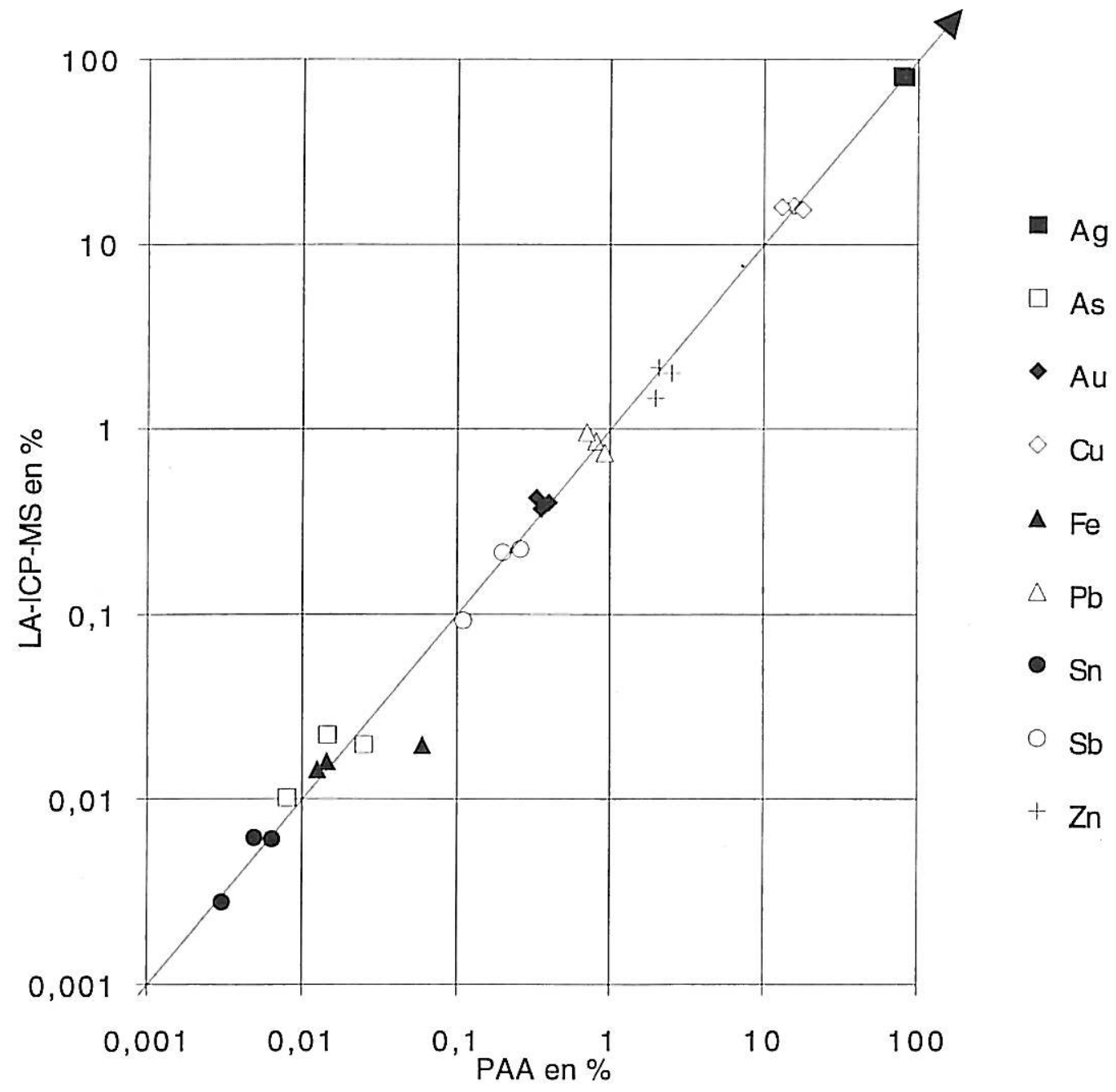

Fig. 8: Comparaison des résultats obtenus par PAA et par LA-ICP-MS pour les pièces d'argent.

métrie de masse couplée aux plasmas induits par hautes fréquences, à de la caractérisation non destructive des archéomatériaux est donc d'ores et déjà envisageable. Cette nouvelle technique associée aux méthodes nu- cléaires permet en effet de disposer d'un outil d'analyse pouvant répondre à un grand nombre de problématiques archéologiques nécessitant des analyses non destructives et ceci, pour la plupart des matériaux archéo- 


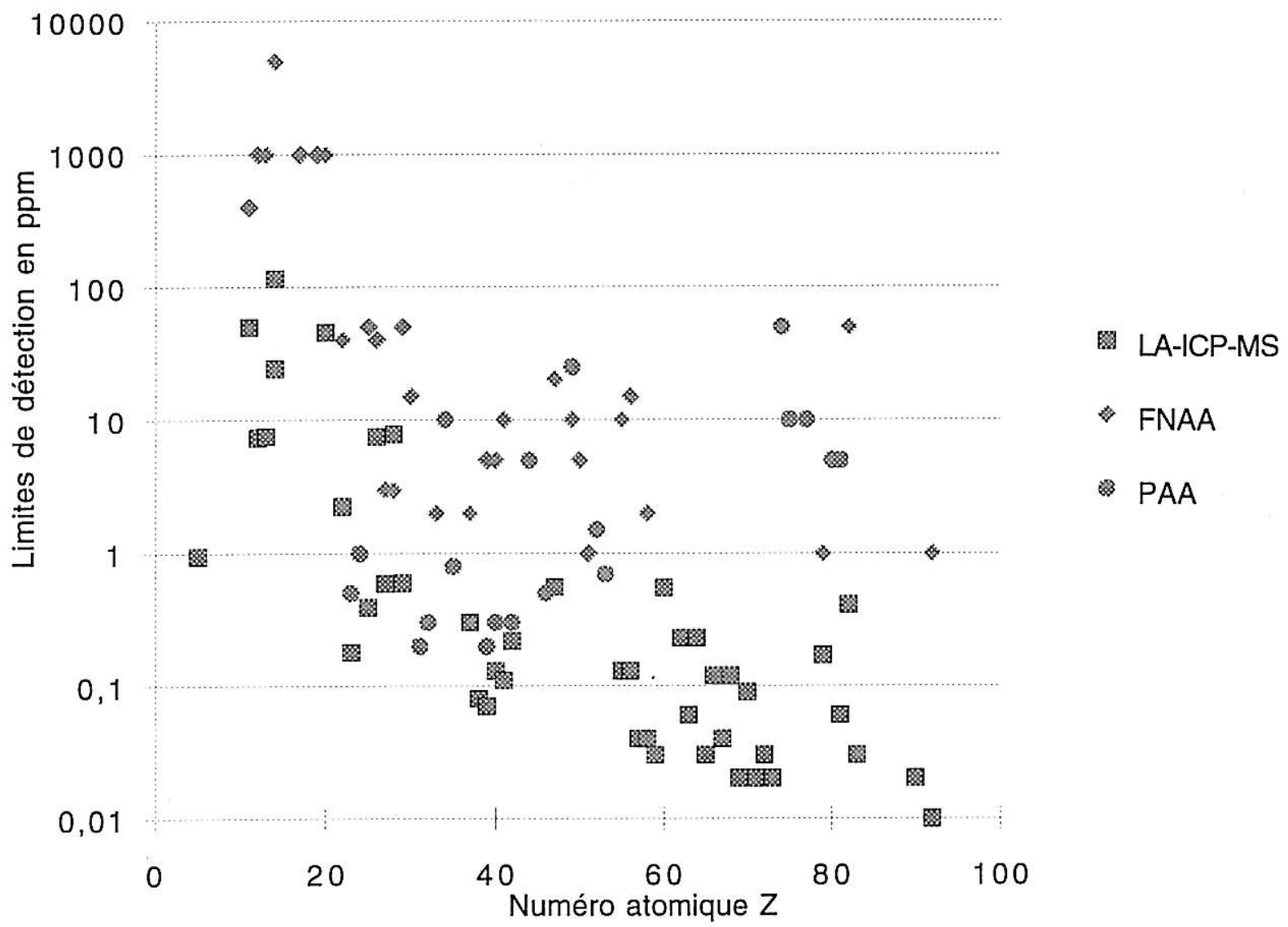

Fig. 9: Comparaison des limites de détection obtenues par FNAA (verres), PAA (or et argent) et LA-ICP-MS.

logiques tant que l'altération de la surface de l'objet n'est pas trop importante.

\section{BIBLIOGRAPHIE}

ARROWSMITH, P., 1987 - Laser ablation of solids for elemental analysis by inductively coupled plasma mass spectrometry, Anal. Chem., 59, 347.

BARRANDON, J.N., 1980 - Méthodes par activation et numismatique, Journal of Radioanalytical Chemistry, 55, 2, 317327.

BARRANDON, J.N., MORRISSON, C., MORRISSON, Ch. et LE ROY-LADURIE, E., à paraitre - The true role of American precious metals transfers to Europe (16th-17th centuries): new evidence from coin analyses, à paraitre dans Post Medieval Archaeology en 1994.

CAUVIN, M.C., BALKAN, N., BESNUS, Y. et SAROGLU, F., 1986 Origine de l'obsidienne de Cafer Höyük (Turquie): premiers résultats, Paléorient, 12/2, 89-97.

FRANCAVIGLIA, V.M., 1990 - Les Gisements d'Obsidienne Hyperalcaline dans l'Ancien Monde: Etude Comparative, Revue d'Archéométrie, 14, 43-63.

GORDUS, A.A., 1972 - Neutron activation analysis of coins and coind streaks in Methods of Chemical and Metallurgical investigation of ancient coinage, E.T. Hall and D.M. Metcalf Ed, Royal Numismatic Society special publication $n$ '8, London, 127-148.

GRATUZE, B., BARRANDON-AL ISA, K. et CAUVIN, M.C.,1993 Non destructive analysis of obsidian artefacts using nuclear tech- niques: investigation of provenance of Near Eastern artefacts, Archaeometry, 35, 1, 11-21.

GRATUZE, B., BARRANDON, J.N., DULIN, L. et AL ISA, K.,1992 Ancient glassy materials analyses: a new bulk non destructive method based on fast neutron activation analysis with a cyclotron, Nucl. Instr. and Meth., B71, 70-80.

GRATUZE, B., SOULIER, I., BARRANDON, J.N. et FOY, D., 1992 De l'origine du cobalt dans les verres, Revued'Archéométrie, 16,97108.

GRAY, A.L. 1985 - Solid sample introduction by laser ablation for inductively coupled plasma mass spectrometry, Analyst, 110, 551.

GUERRA, M.F., BARRANDON, J.N., 1988 - Thermal Neutron Activation Analysis of Archeological Artifacts Using a Cyclotron, Proceedings of the 26th International Archeometry Symposium, Toronto, 262-268.

HOUK, R.S., 1986 - Mass spectrometry of inductively coupled plasma mass, Anat. Chem., 58, 97A-105A.

JARVIS, K.E., GRAY, A.L. et HOUK, R.S., 1992 - Handbook of Inductively Coupled Plasma Mass Spectrometry, Edition Blackie, Glasgow.

KELLER, J. et SEIFRIED, C., 1989 - Obsidian source identification in Anatolia and the Near East, PACT 25, 4, 70-75.

MURR, Missouri University Research Reactor, travaux d'intercomparaison de méthodes, résultats non publiés communiqués par M.D. Glascock.

RENFREW, C. et CANN, J.R., 1964 - The characterization of obsidian and its application to the Mediterranean region, Proc. Prehistor. Soc., 30, 111-133. 